\title{
Trends in colorectal cancer care in southern England, 1989-1993: using HES data to inform cancer services reviews
}

\author{
Allyson M Pollock, Neil Vickers
}

\begin{abstract}
Background-This paper describes trends in hospital activity, hospital admissions, and treatments for colorectal cancer on residents of the South Thames regions (population 8 million) between 1989-1993 against the background of the Calman Report on the future of cancer services in England and Wales.

Methods-The analyses are derived from UK hospital data, which are collected as finished consultant episodes (FCEs). These are defined as episodes "where a patient has completed a period of care under a consultant and is either transferred to another consultant or is discharged." Probability matching was used to derive patient-based records, matching FCEs to admissions. A total of 18542 South Thames residents aged 40-99 were admitted for colorectal cancer between 1 January 1989 and 31 December 1993. Time trends were analysed for procedures, FCEs, admissions, and patient numbers by admission type (ordinary admissions and day case admissions).
\end{abstract}

Results-Between 1989 and 1993 inclusive colorectal cancer admissions doubled (98\% increase $p$ (trend) $<0.0001)$. These admissions were a result of a 6.4 -fold increase in day case admissions and a $41 \%$ increase in ordinary admissions. The proportion of patients having a day case admission rose from $9 \%$ in 1989 to $18 \%$ in 1993 ( $p<0.0001)$. Overall, 2894 (16\%) patients had a day case admission; 1894 of these $(65 \%)$ were also admitted as ordinary admissions. The number of FCEs and admissions per patient rose from 1.37 and 1.28 respectively in 1989 to 2.09 and 1.99 respectively in 1993. FCEs were between $5 \%$ and $8 \%$ higher than admissions over the five years. The number of ordinary (that is, overnight) inpatient admissions per patient rose from 1.23 to 1.41 over the five year period and day case inpatient admissions from 1.25 to 3.45 . Chemotherapy accounted for $50 \%$ of the rise in day case admissions; colonoscopy and sigmoidoscopy were associated with a further $18 \%$. Fourteen per cent of the increase in ordinary admissions was also because of chemotherapy.

Conclusion-The monitoring of site specific trends in admission, treatments, and proceduresw on a population basis should be a core requirement of health authori- ties to inform needs assessment, resource allocation, and service planning. The rise in admissions and chemotherapy treatments have implications for drug costs, laboratory and in patient services, monitoring, and clinical audit.

(F Epidemiol Community Health 1998;52:433-438)

In May 1994 the Expert Advisory Group on cancer reported to the Chief Medical Officers of England and Wales on the future of cancer services. ${ }^{1}$ It anticipated that care will be organised on three levels: while primary care will be the focus of care, the expertise and the management of both common and rare cancers will be concentrated in centres of excellence ("cancer centres") serving populations of at least a million. District General Hospitals that can provide a full range of supportive services (including oncology) will be designated "cancer units." These will treat more common cancers. The report also envisages better integration of primary, secondary, and tertiary care and the development of referral and treatment protocols.

These changes are currently being implemented against the background of the NHS internal market. As the report says: "contracts, as well as the mechanisms for monitoring performance, strongly influence what can be achieved in practice." ${ }^{11}$ At present, most cancer services are purchased through general surgical contracts, but the committee's report and, more recently, a review of contracts in South Thames region, have drawn attention to the inadequacy of current contracts and to the need for site specific contracts "for use as local models to build understanding and gain experience in contracting techniques" (Tera Younger, personal communication).

The hospital data that are used to inform contracts are collected in the form of finished consultant episodes (FCEs). These are part of the minimum contract data set that all acute NHS providers are obliged to collect. FCEs are defined as episodes "where a patient has completed a period of care under a consultant and is either transferred to another consultant or is discharged." ${ }^{2}$ They were introduced to measure resource use in the NHS but, with the advent of the internal market, became the currency for contracts: NHS contracts are based on numbers of FCEs rather than on numbers of patients or admissions or procedures. FCEs have been criticised because they are not patient-based, and consequently do not provide a biographical account of care. They have 
also been criticised for providing only a poor measure of workload. ${ }^{3-5}$

This paper presents HES data relating to colorectal cancer admissions on all South Thames residents between 1989 and 1993 . The aim is to provide baseline planning data on activity, workload, and care patterns for purchasers and providers engaged in implementing the changes proposed in the report of the Expert Advisory Committee.

\section{Methods}

HES data were requested from the Office of Population Censuses and Surveys (OPCS) on all FCEs completed by residents of South Thames Region between 1987 and 1994 with a primary diagnosis of colorectal cancer. Among the data requested on each FCE were: sex, seven digit post code, date of birth, procedure code, ICD-9 code, date of admission, date of episode start, and type of stay. FCEs were indexed by postcode, date of birth, and sex. On the advice of the Office for National Statistics and the Oxford Record Linkage Study, we assumed that FCEs with identical postcodes, date of births, and sex related to the same patient (see discussion). Using date of admission and date of episode start, and patient partial identifiers postcode, date of birth, and sex, it was possible to link FCEs to individuals and individuals to admissions.

\section{BASELINE ANALYSIS}

Using the linkage methods described above the age-sex distribution of all individuals diagnosed as having colorectal cancer was derived. New cases were distinguished from repeat cases (that is, cases previously known to the service).

\section{TRENDS IN CARE}

Admissions

Time trends in FCEs, admissions, and numbers of residents receiving care were analysed using the $\chi^{2}$ test for trend. In all cases, the null hypothesis was that there would be no differences over time and $p<0.05$ was considered significant. The mean numbers of admissions and FCEs per patient were also calculated. Using population estimates supplied by OPCS (in five year age bands for each sex), age standardised admissions rates and age standardised person-based admissions rates (numbers of individuals admitted per 1000 of the population) were derived for each of the five years.

\section{Treatment}

HES data include four procedure variables per FCE. All procedures (from any of the four variables) were noted and ranked by the frequency with which they were given over the five years; the proportion of patients receiving each procedure was also noted. To establish whether admissions had become more complex, the number of procedures recorded for each admission was analysed over time and related to the numbers of people treated. As a quality control measure, the ratio of procedures to patients was analysed over time. The most common procedures were analysed in more detail to look at trends over time.

\section{Results}

EXCLUSIONS

When we matched FCEs to patients, we found that patient numbers doubled in 1989 and stabilised thereafter. We assumed that this doubling was artefactual (it contradicts cancer registry incidence calculations ${ }^{6}{ }^{7}$ ) and that 1989 was the first year with relatively complete ascertainment. Accordingly, data from 1987 and 1988 were excluded. We also excluded data on patients treated in 1994 as we only had data on FCEs occurring between 1 January and 31 March. HES data are processed by financial year (from 1 April to 31 March) rather than by calendar year

Over the five year period 18542 South Thames patients residents completed 33966 admissions and 36196 FCEs. Analysis was confined to FCEs completed by patients aged 40-99 years on admission between 1 January 1989 and 31 December 1993 inclusive.

\section{BASELINE ANALYSIS}

A total of 10127 patients (55\%) were recorded as having colon cancer, 7311 patients (39\%) with rectal cancer only and 1104 patients $(6 \%)$ with tumours of both colon and rectum. Men accounted for a higher proportion of cases among the under 70s (table 1), but in older age groups, women predominated.

TRENDS IN ADMISSIONS, FCES, AND INDIVIDUALS There was a $98 \%$ rise in total admissions from 4737 in 1989 to 9362 in 1993 (p (trend) < $0.0001)$, based on a $640 \%$ increase in day case inpatient admissions and a $41 \%$ increase in ordinary (overnight) inpatient admissions (table 2). A highly significant trend is also seen for FCEs ( $\mathrm{p}$ (trend) $<0.0001$ ) from 5105 in

Table 1 Total numbers of patients analysed; by age, sex, and primary tumour site

\begin{tabular}{|c|c|c|c|c|c|c|c|c|c|}
\hline \multirow[b]{2}{*}{ Age } & \multicolumn{4}{|l|}{ Men } & \multicolumn{4}{|c|}{ Women } & \multirow[b]{2}{*}{ Total } \\
\hline & Colon & Rectum & Both & All (\%) & Colon & Rectum & Both & All (\%) & \\
\hline $40-44$ & 84 & 62 & 16 & $162(2)$ & 63 & 63 & 18 & $144(2)$ & 306 \\
\hline $45-49$ & 118 & 97 & 38 & $253(3)$ & 121 & 94 & 26 & 241 (3) & 494 \\
\hline $50-54$ & 200 & 183 & 43 & $426(5)$ & 180 & 130 & 37 & $347(4)$ & 773 \\
\hline $55-59$ & 330 & 286 & 70 & $686(8)$ & 362 & 201 & 53 & $616(7)$ & 1302 \\
\hline $60-64$ & 510 & 459 & 83 & $1052(12)$ & 493 & 315 & 79 & $887(9)$ & 1939 \\
\hline $65-69$ & 781 & 626 & 107 & $1514(17)$ & 712 & 456 & 87 & 1255 (13) & 2769 \\
\hline $70-74$ & 804 & 657 & 73 & $1534(17)$ & 852 & 556 & 81 & 1489 (16) & 3023 \\
\hline $75-79$ & 821 & 692 & 82 & 1595 (18) & 970 & 656 & 69 & 1695 (18) & 3290 \\
\hline $80-84$ & 630 & 491 & 53 & $1174(13)$ & 939 & 540 & 55 & $1534(16)$ & 2708 \\
\hline $85+$ & 356 & 307 & 12 & $675(7)$ & 801 & 440 & 22 & $1263(13)$ & 1938 \\
\hline Total & 4634 & 3860 & 577 & $9071(100)$ & 5493 & 3451 & 527 & $9471(100)$ & 18542 \\
\hline
\end{tabular}


Table 2 FCEs, admissions, and patient numbers (new cases and repeat cases combined); by type of stay and year of admission

\begin{tabular}{|c|c|c|c|c|c|c|c|c|c|}
\hline & \multicolumn{3}{|c|}{ Finished consultant episodes } & \multicolumn{3}{|l|}{ Admissions } & \multicolumn{3}{|c|}{ Total patients admitted } \\
\hline & Ordinary & Day cases & Total & Ordinary & Day cases & Total & Ordinary & Day cases & Total $^{\star}$ \\
\hline 1989 & 4655 & 450 & 5105 & 4289 & 448 & 4737 & 3492 & 359 & 3713 \\
\hline 1990 & 5045 & 755 & 5800 & 4640 & 745 & 5385 & 3714 & 483 & 4025 \\
\hline 1991 & 5830 & 1228 & 7058 & 5417 & 1225 & 6642 & 4044 & 656 & 4395 \\
\hline 1992 & 6406 & 1978 & 8384 & 5866 & 1974 & 7840 & 4274 & 825 & 4740 \\
\hline 1993 & 6508 & 3327 & 9835 & 6042 & 3320 & 9362 & 4282 & 963 & 4713 \\
\hline Total & 28444 & 7738 & 36182 & 26254 & 7712 & 33966 & 19806 & 3286 & 21586 \\
\hline
\end{tabular}

* Some patients were admitted as both ordinary and day cases; accordingly, the total number of patients as listed in this column is lower than the sum of ordinary and day case patients.

Table 3 Ratios of FCEs to patients, admissions to patients, and FCEs to admissions; by type of stay and year of stay

\begin{tabular}{|c|c|c|c|c|c|c|c|c|c|}
\hline & \multicolumn{3}{|c|}{ FCEs to patients } & \multicolumn{3}{|c|}{ Admissions to patients } & \multicolumn{3}{|c|}{ FCEs to admissions } \\
\hline & Ordinary & Day cases & Total ${ }^{\star}$ & Ordinary & Day cases & Total $^{\star}$ & Ordinary & Day cases & Total ${ }^{\star}$ \\
\hline 1989 & 1.33 & 1.25 & 1.37 & 1.23 & 1.25 & 1.28 & 1.09 & 1.00 & 1.08 \\
\hline 1990 & 1.36 & 1.56 & 1.44 & 1.25 & 1.54 & 1.34 & 1.09 & 1.01 & 1.08 \\
\hline 1991 & 1.44 & 1.87 & 1.61 & 1.34 & 1.87 & 1.51 & 1.08 & 1.00 & 1.06 \\
\hline 1992 & 1.50 & 2.40 & 1.77 & 1.37 & 2.39 & 1.65 & 1.09 & 1.00 & 1.07 \\
\hline 1993 & 1.52 & 3.45 & 2.09 & 1.41 & 3.45 & 1.99 & 1.08 & 1.00 & 1.05 \\
\hline All years & 1.44 & 2.35 & 1.68 & 1.33 & 2.35 & 1.57 & 1.08 & 1.00 & 1.07 \\
\hline
\end{tabular}

*These ratios are calculated using the total number of patients admitted as a denominator, not the sum of ordinary and day case patients.

1989 to 9835 in 1993 (table 2). Day case admissions accounted for $9 \%$ of all admissions and FCEs in 1989 rising to $35 \%$ and $34 \%$ respectively by 1993 ( $\mathrm{p}$ (trend) < 0.0001).

The total number of individuals having at least one ordinary or day case admission rose by $23 \%$ (p (trend) $<0.001$; not shown in a table). The proportion of patients having a day case admission rose significantly from $9 \%$ in 1989 to $18 \%$ in 1993 (not shown in a table). Over the five year period, $2894(16 \%)$ patients had a day case admission, 1894 (65\%) of whom were also admitted as ordinary admissions. (The total listed in table 2 is based on the number of admissions each year. As some patients were admitted in more than one year, they will be counted more than once.) There was no increase in the proportion of patients seen solely as day case admissions - that is, day cases not subsequently or previously admitted as ordinary admissions (not shown in a table).

In 1989, the ratios of FCEs to patients and admissions to patients were 1.37 and 1.28

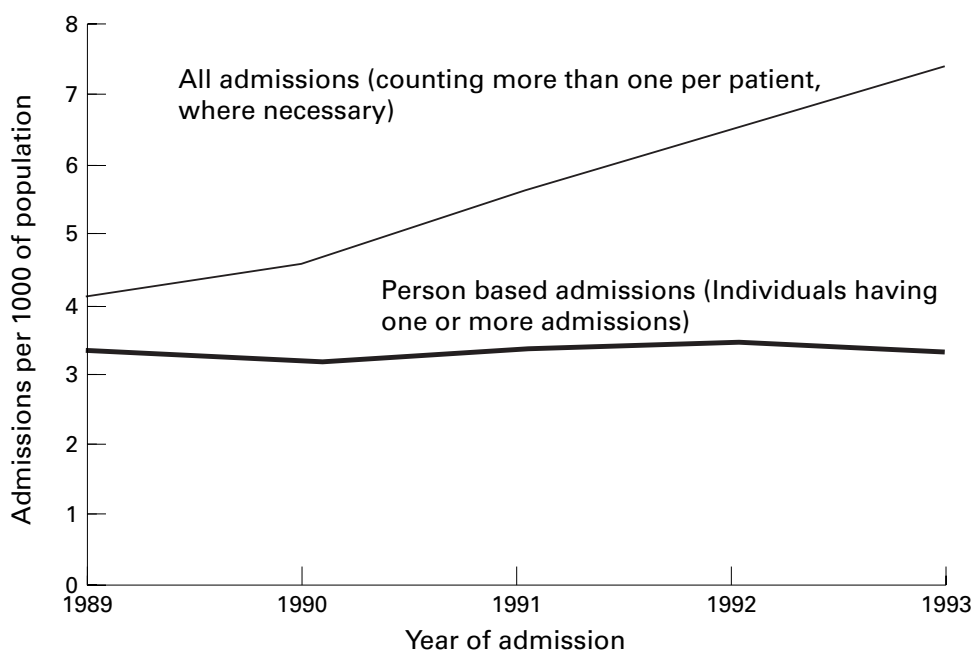

Figure 1 Age standerdised admission rates for colorectal cancer per 1000 of the population: residents of South Thames RHA, 1989-1993. respectively; by 1993 these had risen to 2.09 and 1.99 (table 3). FCEs were between $5 \%$ and $8 \%$ higher than admissions over the five years. For ordinary admissions the number of admissions per patient rose from 1.23 to 1.41 over the five year period while for day cases the ratio of admissions to patients rose from 1.25 to 3.45 .

Population rates

Age standardised admission rates increased at a similar rate to crude admissions (from 4.1 per 1000 in 1989 to 7.4 per 1000 in 1993 ) but the age standardised person-based admission rate remained static at 3.3 per 1000 in 1989 and 3.4 per 1000 in 1993 (fig 1). This latter rate was based on new cases only.

TRENDS IN TREATMENT AND PROCEDURES

A total of 16423 patients (89\%) and 29727 admissions ( $88 \%$ ) had at least one procedure recorded (not shown in a table). Between 1989 and 1993 the proportion of patients and admissions for whom no procedure was recorded fell from $16 \%$ to $8 \%$ ( $p$ (trend) < 0.001 ; not shown in a table). A total of 11453 patients $(62 \%)$ were admitted just once and completed only one FCE (not shown in a table). Table 4 shows the sharp rise in the number of procedures per patient over time.

Table 5 ranks procedures by the numbers of admissions and patients for ordinary and day case admissions respectively for all five years combined. The coding of procedures did not allow us to distinguish curative and palliative procedures from adjuncts to care; because of this, local anaesthetic and blood transfusion appear among the most common procedures.

Procedures for ordinary admissions

Eighteen per cent of ordinary admissions underwent excision of the rectum; a further $11 \%$ were admitted for excision of the right hemicolon. With the exception of 
Table 4 Ratio of procedures to patients; by type of stay and year of admission

\begin{tabular}{|c|c|c|c|c|c|c|}
\hline \multirow[b]{2}{*}{ Year } & \multicolumn{2}{|c|}{ Number \% Procedures } & \multicolumn{2}{|c|}{$\begin{array}{l}\text { Number \% Patients } \\
\text { (new and repeat) }\end{array}$} & \multicolumn{2}{|c|}{$\begin{array}{l}\text { Number \% Procedures to } \\
\text { patients ratio }\end{array}$} \\
\hline & Ordinary & Day case & Ordinary & Day case & Ordinary & Day case \\
\hline 1989 & 5506 & 445 & 3492 & 359 & 1.58 & 1.24 \\
\hline 1990 & 6517 & 748 & 3714 & 483 & 1.75 & 1.55 \\
\hline 1991 & 7121 & 1193 & 4044 & 656 & 1.76 & 1.82 \\
\hline 1992 & 7815 & 1571 & 4274 & 825 & 1.83 & 1.90 \\
\hline 1993 & 8331 & 2292 & 4282 & 963 & 1.95 & 2.38 \\
\hline Total & 35290 & 6249 & 19806 & 3286 & 1.78 & 1.90 \\
\hline
\end{tabular}

Table 5

A Most common procedures recorded for ordinary inpatient admissions

\begin{tabular}{|c|c|c|c|c|}
\hline \multirow[b]{2}{*}{ Procedure } & \multicolumn{2}{|c|}{ Admissions } & \multicolumn{2}{|l|}{ Patients } \\
\hline & Number & $\%$ & Number & $\%$ \\
\hline Excision of the rectum & 4893 & 18.63 & 4815 & 27.45 \\
\hline Other excision of right hemicolon & 2833 & 10.79 & 2826 & 16.11 \\
\hline Colostomy, ileostomy, caecostomy, etc & 2619 & 9.98 & 2440 & 13.91 \\
\hline Blood transfusion & 2312 & 8.81 & 2152 & 12.27 \\
\hline Excision of sigmoid colon & 1869 & 7.12 & 1864 & 10.63 \\
\hline Sigmoidoscopy & 1692 & 6.44 & 1678 & 8.94 \\
\hline Colonoscopy & 1391 & 5.30 & 1299 & 7.40 \\
\hline General anaesthetic & 1248 & 4.75 & 1171 & 6.68 \\
\hline Chemotherapy & 962 & 3.66 & 373 & 2.13 \\
\hline Excision of left hemicolon & 804 & 3.06 & 802 & 4.57 \\
\hline Extended excision of right hemicolon & 673 & 2.56 & 673 & 3.84 \\
\hline Other excision of colon & 471 & 1.79 & 468 & 2.67 \\
\hline Urethral catheterisation of bladder & 387 & 1.47 & 377 & 2.15 \\
\hline \multicolumn{5}{|c|}{ B Most common procedures recorded for day case inpatient admissions } \\
\hline \multirow[t]{2}{*}{ Procedure } & \multirow{2}{*}{\multicolumn{2}{|c|}{ Admissions }} & \multicolumn{2}{|l|}{ Patients } \\
\hline & & & Number & $\%$ \\
\hline Chemotherapy & 2872 & 37.24 & 259 & 9.01 \\
\hline Endoscopy (colorectal) & 2325 & 30.15 & 2030 & 70.64 \\
\hline Anaesthetic & 209 & 2.71 & 172 & 5.99 \\
\hline Unspecified procedure on large instestine & 109 & 1.41 & 106 & 3.69 \\
\hline Endoscopy (upper gastrointestinal tract) & 100 & 1.30 & 99 & 3.44 \\
\hline Unspecified procedure on the bowel & 56 & 0.73 & 55 & 1.91 \\
\hline
\end{tabular}

Table 6 Numbers of patients receiving chemotherapy; by year and type of admission

\begin{tabular}{llllll}
\hline Year & Ordinary & Day cases & Total & $\%$ & $\begin{array}{l}\text { Mean FCEs } \\
\text { per patient }\end{array}$ \\
\hline 1989 & 42 & 7 & 47 & 1.27 & 2.45 \\
1990 & 43 & 28 & 62 & 1.54 & 4.44 \\
1991 & 93 & 56 & 134 & 3.04 & 4.62 \\
1992 & 83 & 68 & 145 & 3.05 & 4.88 \\
1993 & 155 & 146 & 288 & 6.11 & 6.49 \\
\hline
\end{tabular}

${ }^{\star}$ As some patients received chemotherapy as both day case and ordinary admissions, the total in this column is lower than the sum of the rows in the two previous columns.

chemotherapy, there were no significant time trends in either the proportion of patients receiving each procedure or in the frequency with which each procedure was given (not shown in a table). In 1989, chemotherapy accounted for $90(2 \%)$ admissions for $42(1 \%)$ patients. By 1993, these figures had risen to $361(4 \%)$ and 137 respectively $(4 \%)$ (p (trend) $<0.001$ in both cases; not shown in a table). Fourteen per cent of the increase in ordinary admissions were for chemotherapy (not shown in a table).

\section{Day case procedures}

Chemotherapy accounted for $37 \%$ of all day case inpatient admissions. There was a significant increase over time in the number of day case admissions for chemotherapy ( $\mathrm{p}$ (trend) < 0.001 ; not shown in a table). In 1989 , chemotherapy accounted for $32(7 \%)$ day case admissions by 1993 the figure was 1476 (64\%). The number of patients receiving chemotherapy also rose significantly (table 6), although only $9 \%$ of day case patients received it (table 5). The number of day case admissions per patient for chemotherapy rose from 4.57 in 1989 to 11.71 in 1993 (not shown in a table). Day case admissions with a mention of chemotherapy increased from 32 to 1476 while total day case admissions increased from 448 to 3320 . Thus, around $50 \%$ (1444 of 2872 ) of the increase in day case admissions were for chemotherapy.

Colonoscopy and sigmoidoscopy were recorded on $30 \%$ of all day case admissions and given to $71 \%$ of patients (table 5). Colonoscopies rose by $50 \%$ among day case admissions over the five years, from 483 in 1989 to 1025 in 1993. The number of day case admissions for colonoscopy alone (that is, with no other procedure recorded) or with anaesthetic fell from 220 $(49 \%)$ in 1989 to $184(6 \%)$ in 1993 (p (trend) $<0.00001)$. Admissions for sigmoidoscopies only fell from $35(8 \%)$ in 1989 to $59(2 \%)$ in 1993 ( $\mathrm{p}$ (trend) < 0.001). Colonoscopy and sigmoidoscopy were associated with $18 \%$ of the rise in day case admissions.

\section{Discussion}

TRENDS IN ADMISSIONS

This paper shows a striking increase in admissions for colorectal cancer for South Thames residents. Over the five year period 1989-93 inclusive ordinary admissions increased by $40 \%$ and there was a sixfold increase in day case admissions. Day cases accounted for $9 \%$ of FCEs in 1989 rising to $35 \%$ in 1993 . This is at a time when purchasers continue to set contracts in the general surgical contracts on a historical baseline of 1990 .

Four possibilities could account for the rises in FCEs and admissions: (1) they might be a function of increased incidence in the disease either because of changing demographic profiles or because of epidemiological changes; (2) they might reflect more assiduous recording on the part of providers, especially for day case admissions as a result of the internal market; (3) for financial reasons, providers might be increasing the number of FCEs and admissions completed by each patient (gaming); and (4) they might be the result of therapeutic and diagnostic advances increasing the intensity and volume of care.

\section{CHANGES IN INCIDENCE}

The number of new patients remained static over time (in 1989 it was not possible to distinguish new from repeat patients because the 1988 data were of too poor quality and this baseline year will overestimate new patients; fig 1). The person-based admission rate (the annual number of individuals having one admission or more) showed no change over time.

It is not the case therefore that more patients are being treated ${ }^{8}$; rather, it would seem that some patients are having more admissions and are receiving more complex packages of care, for example, adjuvant chemotherapy - that is, chemotherapy combined with a major surgical procedure. The big increases in FCEs and acute and day case admissions over time are 


\section{KEY POINTS}

- Admissions for colorectal cancer in South Thames doubled between January 1989 and December 1993. This was based on a $640 \%$ increase in day case admissions and a $41 \%$ increase in ordinary admissions.

- The number of patients treated remained constant.

- The increases in admissions appeared to be fuelled by a small number of patients receiving more complex packages of care, especially surgery with adjuvant chemotherapy.

because of a relatively small proportion of patients being admitted to receive more procedures more frequently (tables 5 and 6 ).

\section{RECORDING PRACTICE}

The increase in the number of procedures per patients suggests that providers may have become more assiduous in recording procedures. The advent of NHS contracting in 1991 gave providers an incentive to improve recording practices over time. There might also have been a trend away from recording some procedures as outpatient admissions towards day case admissions; again, chemotherapy administered in wards or in outpatient departments might formerly not have been counted. As we did not have outpatient activity data we could not investigate this possibility.

ARE PROVIDERS MAXIMISING THE NUMBER OF FCES COMPLETED BY EACH PATIENT?

The ratio of FCEs to admissions remained constant over the five year period (from 1.08 to 1.05). This suggests that providers are not gaming by increasing the number of FCEs per admission, in contrast with some of the evidence reported in the literature.

THERAPEUTIC AND DIAGNOSTIC ADVANCES Although survival from colorectal cancer has remained static over the past 20 years, advances have been made in diagnostic and chemotherapeutic interventions for some subgroups. The sixfold rise in day case admissions is strongly associated with chemotherapy. Nine per cent of patients admitted as a day case for chemotherapy generate $46 \%$ of all day case procedures. Much of the remainder of the rise in day cases seems to be because of colonoscopies and sigmoidoscopies.

While these increases may be artefactual, there is a trend to more adjuvant chemotherapy reported in the literature. The Levamisole and 5-FU trials have shown a survival benefit for rectal cancer Dukes's stage C patients. ${ }^{9}$ Recent years have witnessed the introduction of new chemotherapeutic agents for the treatment of stage B and C colorectal cancers and a number of trials including the national QUASAR trial have been launched. It is probable that a similar trend would be seen for radiotherapy; however radiotherapeutic proce- dures are not coded in the Classification of Operations. Some GPs and consultants now run screening programmes. These, together with open access clinics for primary care, may have increased the investigation rate. These are areas that purchasers need to look at in more detail.

DATA QUALITY

Two criticisms are commonly advanced against the use of HES data: that the quality of the data is poor and that the form in which they are presented, the FCE, is not helpful because it provides neither a description of the hospital spell nor a biographical account of care.

The Department of Health has undertaken studies to estimate the completeness of HES coverage and the reliability of diagnosis. Overall coverage has been consistently high. For the financial year 1989-90 it was estimated at 98\% and the figures for each subsequent financial year were $102 \%$ (because of extra submissions from a single RHA), $97 \%$, 99\%, and $99 \%(\mathrm{Mr}$ David Hewitt, DoH, personal communication). The percentage of cases in each year with a reliable diagnosis was $84 \%, 86 \%, 95 \%, 94 \%$, and 94\% (Mr David Hewitt, DoH, personal communication). In $198985 \%$ of all admissions had a procedure recorded; by 1994 this had risen to $92 \%$. Walshe et al found only $68 \%$ agreement for three digit ICD code in a study comparing HES data and a clinical coding system relating to a patients attending a South Thames urology clinic. ${ }^{11}$ Panayiotou examined HES data in Walsall and found $75 \%$ of Körner codes tallied absolutely with clinical casenotes and that a further $19 \%$ were partly correct. ${ }^{12} \mathrm{~A}$ recent study of HES data for two North Thames hospital has found much poorer coding (J Dixon, personal communication). It is probable that the quality of data is improving but further validation studies are required. The ratio of emergency to elective admissions remained constant over time, which suggests some homogeneity. We were unable to estimate the reliability of the data with which we were supplied.

\section{PATIENT-BASED DATA}

We were able to go some way towards distinguishing new patients from those previously in contact with the service. (With more data from previous years, it would have been possible to do so more accurately.) We could not distinguish readmissions from admissions for new events in repeat admissions, as this information is not collected.

The pitfalls associated with our methods of patient linkage have been discussed by Gill et $a l .{ }^{13} \mathrm{It}$ is subject to three main sources of error. The first stems from the assumption that records with the same postcode, date of birth, and sex relate to a single patient. Given the large numbers of birth dates in our sample, and the large numbers of postcodes and the incidence of colorectal cancer in the population as a whole, the probability of this assumption being false is very low. A more likely source of error is coding error. If there is any inconsistency in the recording of the index variables, our 
method will assign the FCE to another patient record and this would lead to overestimation of patient numbers. To estimate the scope for error arising from small errors in the coding of the index variables we reindexed the database, using four digit postcode and year of birth (instead of seven digit postcode and full date of birth). We also linked cases without using patient sex as an index variable. We lost 19 cases using four digit postcode, full date of birth, and sex; four cases using full postcode, year of birth, and sex; and four cases using full post code and full date of birth only. Lastly, our method of record linkage makes no allowance for patients moving home between admissions. This could lead to an overestimate of new patient numbers. To get a rough estimate of how many people might have moved, we consulted the 1991 census, which records the number of people resident at a different address exactly one year before enumeration. A total of 65615 of 2551090 (2.6\%) South Thames residents aged 45-99 (the type of data aggregation used in the census did not permit us to take data on those aged 40-99) had changed address between 1990 and 1991 . Applying this percentage annually to our new cases over the five years, we find an overestimate of 561 person-based admissions (561 of $18542=3.0 \%$ of our sample).

None of these sources of error (either on its own or in combination) could account for the magnitude of the rises reported here in admissions and FCEs.

\section{Conclusions}

These data show the value of site specific analysis for planning cancer services and for setting contracts. Providers have yet to demonstrate that the government's aim of treating more patients as day cases can be achieved for colorectal cancer. The number of new patients being treated solely through day case admissions remained static (fig 1) and the small rise in ordinary admissions suggests that no further shift to day case work is probable in the near future. However, both inpatient and day case activity looks set to grow further as a result of chemotherapeutic advances and screening and this has serious implications for contracts. It remains to be seen whether the trends we have described will continue.

The growth in day case procedures (especially chemotherapy) for colorectal cancer has many resource implications that purchasers will need to consider: these include drug costs, laboratory services, and inpatient facilities. At present, most contracts for cancer services are subsumed into general surgery or oncology services: there has been little detailed analysis by disease site of the resource implications across specialties or providers. As radiotherapy is a important cancer treatment; its omission from the HES dataset creates a serious difficulty in monitoring patient care. This omission needs to be resolved urgently. We are currently undertaking further work to model resident flows and provider work loads. The changes in patterns of resource use revealed by this analysis fully justify the Expert Advisory Committee's call for more detailed, site specific contracting in the future.

Funding: South West Thames Region Research and Development Programme.

Conflicts of interest: none.

1 Expert Advisory Group on Cancer. A policy framework for commissioning cancer services. Leeds: NHSME, 1994.

2 Government Statistical Service. Hospital episode statistics, 1989-1990. London: HMSO, 1993.

3 Radical Statistics Health Group. NHS reforms: the first six Radical Statistics Health Group. NHS reforms: the first six
months - proof of progress or a statistical smokescreen? months - proof of progr

4 Clarke A, McKee M. The consultant episode: an unhelpful measure. BMF 1992;305:1307-8.

5 Pollock AM, Majeed A. Consultant episodes. BM7 1993; 306:141-2.

6 Thames Cancer Registry. Cancer in South East Thames 1987-1989. Sutton, Surrey: Thames Cancer Registry, 1992.

7 Thames Cancer Registry. Cancer in South West Thames 987-1989. Sutton, Surrey: Thames Cancer Registry, 1992.

8 Aylin P, Majeed FA, Cook DG. Home visiting by general practitioners in England and Wales. BMF 1996;313:20710.

9 Majeed FA, Cook DG. Age and sex differences in the management of ischaemic heart disease. Public Health 1996; 110:7-12.

10 Majeed A. The basis of a more rational method of funding primary medical care? BMF 1996;312:1012-13.

11 Majeed FA, Martin D, Crayford T. Deprivation payments to general practitioners: limitations of census data. BMF 1996;313:669-70.

12 Sloggett A, Joshi H. Higher mortality in deprived areas: community or personal disadvantage? BMf 1994;309: $1470-4$

13 Gill L, Goldacre M, Simmons H, et al. Computerised linking of medical records: methodological guidelines. F Epidemiol Community Health 1993;47:316-19. 